\title{
Development of an Arabic Food Composition Database for an Arabic Online Dietary Assessment Tool: myfood24
}

\author{
A. O. Bawajeeh ${ }^{1-2}$, S Kalendar ${ }^{1-3}$, G. Scarpa ${ }^{4}$, N. Hancock ${ }^{5}$, S. Beer ${ }^{6}$, L. Gibson $^{6}$, G. Williams ${ }^{6}$, \\ B. Dashti ${ }^{7}$, S. Albar ${ }^{2,8}$, H. Ensaff ${ }^{1}$, M. A. Zulyniak ${ }^{1}$, C. E. Evans ${ }^{1}$ and J. E. Cade ${ }^{1}$ \\ ${ }^{1}$ Nutritional Epidemiology Group, School of Food Science and Nutrition, University of Leeds, Leeds LS2 9JT, UK, \\ ${ }^{2}$ Department of Food and Nutrition, Faculty of Human Sciences and Design, King Abdulaziz University, Jeddah 3270, \\ Saudi Arabia, \\ ${ }^{3}$ Public Authority for Applied Education \& Training, Kuwait, \\ ${ }^{4}$ School of Earth and Environment and School of Food Science and Nutrition, \\ University of Leeds, Leeds LS2 9JT, UK, \\ ${ }^{5}$ School of Medicine, University of Leeds, Leeds LS29JT, UK, \\ ${ }^{6}$ Dietary Assessment Ltd., UK, \\ ${ }^{7}$ Kuwait Institute for Scientific Research, Kuwait and \\ ${ }^{8}$ Saudi Food and Drug Authority, Northern Ring Branch Road, Dist. Unit number: 14904 Dist. \\ Riyadh 6336, Saudi Arabia
}

Assessing dietary intake requires comprehensive food composition databases (FCDB), supporting linkage between foods and nutrients ${ }^{(1)}$. While traditional methods of collecting dietary intake have been interviewer-led, web-based systems offer advantages allowing for self-administered recalls. In light of this, currently available FCDB for Gulf Cooperation Council countries is limited ${ }^{(2)}$, and an Arabic web-based dietary assessment tool is not yet available. We aimed to develop an Arabic online dietary assessment tool, myfood $24^{(3)}$ with a new Arabic FCDB, the initial focus will be foods from Saudi Arabia and Kuwait.

Food information was sourced from existing Middle Eastern food composition tables, research articles, and Back-of-pack (BOP) nutrient labels, with the addition of generic foods from the UK Composition of Foods Integrated Database (CoFID). An initial cleaning involved the removal of duplicate foods and reformatting of units for some nutrients. Recipe ingredients and foods were mapped to similar generic items in the CoFID database to generate the missing nutrient data. Multiple options for portion size estimations were assigned (e.g. images, household measurements, serving sizes and average portion sizes). Then, the entire database was translated into Arabic and quality checked.

The new Arabic FCDB contains 2016 food items with complete macronutrient and micronutrient data $(120$ nutrients). $79 \%$ ( $=$ 1585) were generic items from the UK CoFID, $13 \%(n=271)$ were from BOP data labels for local and regional branded products in Saudi Arabia and Kuwait and $8 \%(n=160)$ were composite food items from existing published sources. Seventy-seven new recipes were created; 64 recipes were already available from Kuwait. Examples of local recipes with full nutritional information include marag laham (mutton stew); makbous laham (rice with lamb); spinach pastry; date cake. The database includes 14\% from both meat products and dishes $(n=286)$ and vegetables and vegetable-based dishes $(n=278) ; 11 \%$ of cereal products and associated dishes $(\mathrm{n}=230) ; 9 \%$ sweet-tasting foods (e.g. desserts and chocolate) $(\mathrm{n}=187)$; and $8 \%$ for fruits $(\mathrm{n}=171)$, seafood $(\mathrm{n}=166)$, and dairy products $(n=166)$. There are only $5 \%$ poultry products and associated dishes $(n=99), 5 \%$ beverages $(n=93)$, and $3 \%$ bean-based dishes $(\mathrm{n}=68)$, while $13 \%$ come from miscellaneous foods including spices $(\mathrm{n}=81)$, savoury snack $(\mathrm{n}=53)$, sauces $(\mathrm{n}=52)$, ready-to-eat products $(n=39)$ and oils $(n=24) .30 \%$ of foods have photographic portion size images in addition to the other options of portion size estimation.

This is the first Arabic FCDB containing 2016 composite dishes, branded and generic foods available for Saudi Arabia, and Kuwait, which will be integrated into an Arabic myfood24, producing the first Arabic online dietary assessment tool. This tool will be important to support the assessment of dietary intake of Arabic-speaker populations. We plan to expand this FCDB to cover more foods from other countries in the Middle East region.

\section{References}

1. Foster E, Hawkins A, Delve J et al. (2014) J. Hum. Nutr. Diet 27, 26-35.

2. Al-Faris N (2017) J. Food Nutr. Res 5, 168-75.

3. Carter M, Albar S, Morris M et al. (2015) Nutrients 7, 4016-32. 